\title{
Assessment of 12 Months Retention in Care among Children living with HIV in a Pediatric Reference Hospital, Yaounde Cameroon
}

\author{
Article by Andreas A. B. Frambo ${ }^{1}$, Francis Ateba. Ndongo ${ }^{2}$ \\ ${ }^{1}$ Public Health, Texila American University, Cameroon \\ ${ }^{2}$ Pediatric Reference Hospital, Yaounde Cameroon, Université de Paris Sud, France \\ E-mail: besong05@yahoo.ca ${ }^{1}$,aframbo05@gmail.com ${ }^{1}$,atebfranck@gmail.com ${ }^{2}$
}

\begin{abstract}
Poor retention in HIV care is a known main challenge for antiretroviral treatment programs resulting to poor performance and increased morbi-mortality. Many studies related to retention are focused on adults including pregnant women, leaving out children, especially in sub Saharan Africa. The objective of this study is to assess 12 months retention and determine factors associated with retention among children on ART. A cross-sectional study was carried out in a pediatric reference in Yaounde, Cameroon. Registers of children enrolled on antiretroviral therapy from Oct 2015 to Oct 2016 were reviewed for retention. Factors associated with retention in children were determined for future improvement.

A total of 416 children were enrolled and after 12 months follow up, 398 (90.9\%) were found to be alive and still on treatment. Young age (age less than 1 year) of start on ART and CD4\% were the factors associated with poor retention \& attrition at 12 months after initiation.

Twelve months retention in care for children living with HIV is near optimal (90.0\%) with a high chance of long term survival. Age less than 1 year and CD4\% were baseline risk factors for poor retention. Given that children rely on their caregivers for optimal retention, there is need for robust prevention of mother to child transmission programs and pediatric case finding \& linkage to care strategies. Quality data will help improve strategies and procedures to boost long term retention required for viral suppression needed end the HIV pandemic.
\end{abstract}

Keywords: HIV, Assessment, Retention, 12 months, Children, Cameroon.

\section{Introduction}

Poor and suboptimal patient retention in HIV care undermines AIDS programmes and patient outcomes, including achieving sustained viral suppression required for epidemic control. The global effort to increase the number of people on ART should also ensure that people taking antiretroviral therapy (ART) are retained in appropriate chronic care for the rest of their life if the chain of HIV infection needs to be effectively cut. Retention in HIV/AIDS programmes is a major challenge in rural and urban settings and across all populations, specifically pediatric and adolescent populations with maturing immune systems and also postpartum women and men in general. According to WHO, multiple factors have been known to play varying roles in loss to follow-up and suboptimal retention including far distance to health facilities, lack of transport, inability to cover for travel expenses, stigma (both from health personnel and non-health personnel) disclosure-related issues, being too sick and lack of understanding of the need for lifelong care and also the poor attitude of health care providers towards people living with HIV (WHO, 2016). These factors are responsible both for adult and pediatric suboptimal retention but none of these is specific for children who solely depend on adults in most of the cases for their own retention to be optimal.

\section{Problem statement}

It has now been shown worldwide that poor retention of patients in HIV care, particularly in the period between testing and starting of ART and after starting ART, is a major driver of the observed poor performance and increased morbidity and mortality related to HIV/AIDS implementation projects (Fox \& Rosen, 2010). In a prospective study on retention and the risk factors for attrition in a large ART public health program in Myanmar, $84 \%$ of patients were retained while $7 \%$ were lost to 
follow up and $9 \%$ had died. In this same study the retention rates of participants at $12,24,36,48$ and 60 months were $86,82,80,77$ and $74 \%$ respectively and tuberculosis (TB) treatment at ART initiation, a prior ART course before program enrollment and literacy were significant predictors for retention in the program(Thida et al, 2014). In another retrospective cohort study of patients initiating outpatient care at the University of Alabama revealed that worse retention in the first 2 years was associated with younger age, higher baseline CD4 count, and substance abuse (Ulett et al, 2009).

In sub-Saharan Africa (SSA) where close to 90\% of PLWHIV are resident, the main reason why retention in HIV care is poor and in some settings very poor is the late identification and very late start of ART often with very low CD4 counts. HIV infected people were usually identified and they unfortunately had to wait for an eligibility before they are started on ART (Roura et al, 2009). Other reasons for poor retention which have been identified include issues of disclosure including fear of being recognized as a client of an HIV clinic, stigmatization and discrimination that could potentially result in many social problems including job loss, partner rejection, social isolation and discrimination. Patient mobility due to factors such as severe illness, distance to clinic, lack of transportation and poverty are also well documented reasons for poor retention in resource limited settings. Some of the factors that account for poor retention are health facility related and include but are not limited to long waiting time at facilities, poor attitude of health workers and lack of good organization (Dahab et al, 2008). It is now known that clients who present at the ART clinic with low CD4 counts are likely to die before reaching stage of ART eligibility. This is the main reason behind the latest recommendation from WHO to start treatment once identified HIV positive (Test and Treat).

\section{Justification and objective}

Identifying patients who are not retained in HIV care can be very challenging as poor retention can include a range of behaviors such as missing a single scheduled clinical visit to lost-to-follow-up (LTFU), a term use to describe patients who fail to present to clinic for more than 90 days. Although overall treatment adherence among sub-Sahara African patients has been high, recent evidence suggests that a large number of PLHIV in the region who have started in treatment programs are not retained in care. A review of 33 patient cohorts taking ART in 13 African countries suggested only 60 percent of patients remain enrolled in programs after two years, with LTFU accounting for 56 percent of all attrition (Rosen et al, 2007 ). In a similar study in Uganda in 2009, over 25 percent of patients eligible for ART did not complete screening and/or begin treatment (Amuron et al, 2009). The incidence rate of LTFU in a cohort study done in South Africa on the determinants of loss to followup in patients on antiretroviral treatment in 2014 was 103 per 1000 person-years in the first year on ART and increased to 405 per 1000 person-years in the eighth year of taking ART. Associated factors related to becoming LTFU included not having a committed partner, being self-employed, detectable last known Viral Load (VL) and a last known World Health Organization clinical stage III or IV (Mberi et al, 2015). Another study on Factors Influencing Retention in Care after Starting Antiretroviral Therapy in a Rural South African Programme revealed that $82.4 \%$ were in active care (including those that had transferred themselves elsewhere) at the end of 6 months, $11.1 \%$ had died and $6.5 \%$ were lost to follow-up (Boyles $e t$ al, 2011). It is for these reasons that we coined this study to assess retention among children living with HIV in a reference pediatric HIV care and treatment center in the capital city of Cameroon.

\section{Methods}

\section{Site selection}

We conducted a descriptive cross-sectional study in the Centre Mere et Enfant/ Fondation Chantal Biya in Yaounde, one of the reference pediatric HIV care and treatment centers in Cameroon. This health facility is also one of the tertiary health institutions in Yaounde, Center region of Cameroon and is focused on managing childhood diseases. The target population in this study was all children less than 19 years of any sex, living with HIV and currently enrolled into HIV care and treatment registry at the reference pediatric HIV care and treatment unit. Registers were used for data collection for one month targeting October 2015 to October 2016. 


\section{Sampling and data collection}

The sampling method was continuous, consecutive and exhaustive. The sample size was determined by the sum of the number of enrolled children in each cohort from October 2015 to October 2016 inclusive. The data collection tool was a standardized questionnaire that captured basic demographic data including age, sex, and caregiver type \& phone number. It also captured data on baseline biological including weight and age of start of ART, the ART regimen, initial CD4 count, current ART status and client retention status. The questionnaire was pretested for 1 week and validated at a different nearby ART clinic to ensure that variables are well understood and captured appropriately. Data was collected in 13 cohorts as it appeared in the ART registers and each cohort was evaluated for 12 consecutive months only. We conducted the data collection procedure and simultaneous data entry and cleaning over a period of two weeks. Data collection was carried out by health care workers who are responsible for filling out registers in the clinic so as to minimize data collection procedures.

\section{Data analysis}

All data collected was entered into a pre-established make view page programmed to flag missing and out of range values so that all data that was not correctly recorded was identified and managed appropriately. Demographic data including age at start of ART was disaggregated by age group and sex and ART regiment was disaggregated into first or second line. Client ART and retention outcomes in care were assessed as "in care"; "loss to follow-up (LTFU)" "discontinued care", "died" and "unknown". LTFU was defined as children who missed their appointments for up to three months and there was no documentation of the outcome after several trials to reach to the client. EPI info version 6.3.2 and Microsoft Excel 2010 were used for data analysis and production of tables and figures. The analysis trend for clinical and retention outcome data as well as their association with treatment outcomes was computed with the help of these software. Retention was computed as the ratio of the number of children found alive after 12 months of ART initiation (including those who were alive and on ART after transfer out) and the number of children initially enrolled on ART. Demographic factors of children who were not retained for up till 12 months were sorted and analyzed. Fischer's exact test was used to determine factors associated with retention and a p-value of less than 0.05 was considered as statistically significant after considering $95 \%$ confidence intervals.

\section{Results}

\section{Demographic and biological characteristics}

A total of 435 children had enrolled on ART at the health facility from the 13 monthly cohorts as of October 2015 to October 2016 among whom 416 were retained for this study. Majority of the enrolled children $238(57 \%)$ were female and $178(43 \%)$ were males. The age range at start of ART between the cohorts was relatively late ranging from 3 months and 8 years with median age being 53 months (IQR, 24-96). The disaggregated age range with the highest frequency was the age 5 to 9 years, representing one-thirds $(30 \%)$ of the total number of children enrolled and currently on ART. The main caregivers recorded during this study were parents $(71 \%)$ followed by grandparents $(16 \%)$. All of the clients assessed in this survey were initiated on a first line regimen. WHO clinical staging was no longer used as a criterion for initiation during the study period as such was not assessed and therefore not recorded in the ART register. Among all the clients enrolled in this study, 373 (89.6\%) had a documented CD4 value or CD4\% at initiation which was used for analysis. Of those with documented CD4 at initiation, the median CD4 value was $14 \%$ (IQR, 11-19, $\mathrm{n}=156$ ) for children less than 5 years and 241 cells/ml (IQR, 201- 264, $\mathrm{n}=217)$ in children greater than 5 years. During ART initiation $301(72 \%)$ of children had a documented associated illness in addition to HIV infection, the majority among which was malnutrition (34\%), followed by skin infestations (23\%) and respiratory tract infections (20\%). Table 1 summarizes the demographic and baseline biological characteristics recorded during this study. 
DOI: $10.21522 /$ TIJPH.2013.05.04.Art020

ISSN: $2520-3134$

\section{Retention after 12 months}

Among the 416 children assessed during this study, 139 (33.4\%) attended the clinic less than 4 times while $184(44.2 \%)$ had between $4 \& 8$ documented clinical visits and 93 (22.4\%) had visited the clinic between 9 and 12 times annually (Figure 1). Among them, 378 (90.9\%) were retained at 12 months after ART initiation. The rate of retention per monthly cohort was lowest for the months of December 2016 and July 2017 with retention rates at 77 and 75 percent respectively. Figure 2 shows the outcome of clients that were assessed for retention 12 months after initiation.

\section{Factors Associated with retention}

Gender was not statistically significant with retention at 12 months after initiation (p-value 0.518 ). Young age was associated with poor retention ( $\mathrm{p}$ 0.039) and children less than 1year old seemed to be at a higher risk of not being retained for up to 12 months. Retention seemed to increase with age; it was lowest in age $<1$ year $(53.8 \%)$ and highest in age range 5-15 years $(87.6 \%)$. There was no significant difference in retention among children with different caregiver types. Higher retention was observed in patients who had a CD4\% (p value 0.049 ) as opposed to those who had a CD4 count. The presence of an illness at the start of ART did not happen to influence the retention after 12 months of initiation in this study. LTFU also occurred at higher rates in younger children and children less than one year old had a higher loss to follow-up rate. Higher mortality rates $(63 \%)$ were observed more within the first six months of ART initiation and 50\% of the death cases were both from under 5 and over 5 years old. All of the 7 clients that discontinued ART were adolescents of the age group 15-19 years.

\section{Discussions}

It is well known that retention in HIV care is vital both to reduce HIV-related mortality and morbidity and as a means to deliver "positive prevention" interventions aimed at reducing ongoing transmission. In this study of retention among children living with HIV in a tertiary health facility in the Central region of Cameroon, over $90 \%$ of children who initiated ART were retained in HIV care 12 months after initiation. This was very similar to findings from South Africa (Sengayi et al, 2013) which showed $90 \%$ retention after 12 months of follow-up in 2013. Studies from across Africa, estimated a median pediatric retention in care for 12 months after treatment initiation to be $82.6 \%$, with a range of 71-95\% (Abuogi et al, 2016). Although some studies differ in the follow-up periods, the rates of retention are fairly similar our study. This suggests different comparable rates of retention across Africa despite nearly similar settings. The above high rates of retention in children within the first twelve months of ART initiation could be as a result of the availability of optimal pediatric products, particularly the fixed dose combinations of pediatric ARVs that are suitable for children to take and provides very limited side effects.

In our analysis, poor retention was associated with young age with a p value of 0.039 . Retention increased proportionately with age, lowest in children with age $\leq 1$ year $(52.9 \%)$ and highest in children with age $>5$ years $(89.3 \%)$. Similar studies in India (NACO, 2011) showed that younger adults fail to access health services efficiently. The WHO report on retention in 2011 stated that, diagnosing and retaining HIV-exposed and infected children and adolescents in care present unique challenges (WHO, 2012). The fact that children are solely dependent on a caregiver for their treatment and retention could be the main reason behind this finding. The CD4\% count was statistically significant in our study with P-value of 0.049. This is consistent with studies by Lessells et al. and Charurat et al. However in a study by Charurat et al. retention was rather lower in baseline CD4 \% when compared to our study. Alvarez-Uria et al. in 2013 demonstrated that was no significant association between baselines CD4 and retention in their study carried out in India. This difference is relation to CD4 could imply that other factors do interfere with CD4 in the attainment of retention in children.

We did not find a significant association between retention and gender in this study. While female children were slightly more than the male participants in this study (57\%) they had an insignificant retention compared to the male counterparts. This has not been the case in other studies which have rather revealed that there is a higher probability for women to be retained in HIV care and treatments 
projects and programs when compared to their male counterparts (Remien et al, 2010). The finding that higher proportion of women initiated on ART remained in follow-up, may reflect genderdifferences in health-seeking behaviors which has been shown to affect retention in care in other resource-limited countries (Forster et al, 2008 \& Basset et al, 2010). We also did not assess for WHO staging because at this time the country had migrated to the test and treat strategy hence the only criterion for eligibility is being HIV positive.

\section{Conclusion}

In this study we conclude that retention in care for children living with HIV at 12 months after initiation is near optimal (90.0\%) and thus there is a high chance of long term survival. Age less than 1 year and CD4\% were baseline risk factors for poor retention. This underscores the need for early diagnosis and prompt initiation of ART before the appearance of signs and symptoms of AIDS defining illnesses (immunodeficiency) in children. This will require very robust prevention of mother to child transmission programs as well as strong pediatric case finding and linkage to care strategies. The challenge with pediatric retention is embodied in adult care since children rely on their caregivers for optimal retention.

It could be recommended that additional resources is needed for the creation of more reference centers for pediatric HIV care and treatment as this will maximize pediatric retention, given the child friendly environment. With such centers, the prevention of mother to child transmission will be made more robust and this will enhance pediatric case finding and improve linkage to care required for early start of treatment. Quality data management in pediatric HIV programs will help improve strategies and procedures to boost long term retention. This will enable the possible attainment of the third 90 of viral suppression as the struggle to end the HIV pandemic is ongoing.

\section{Figures and tables}

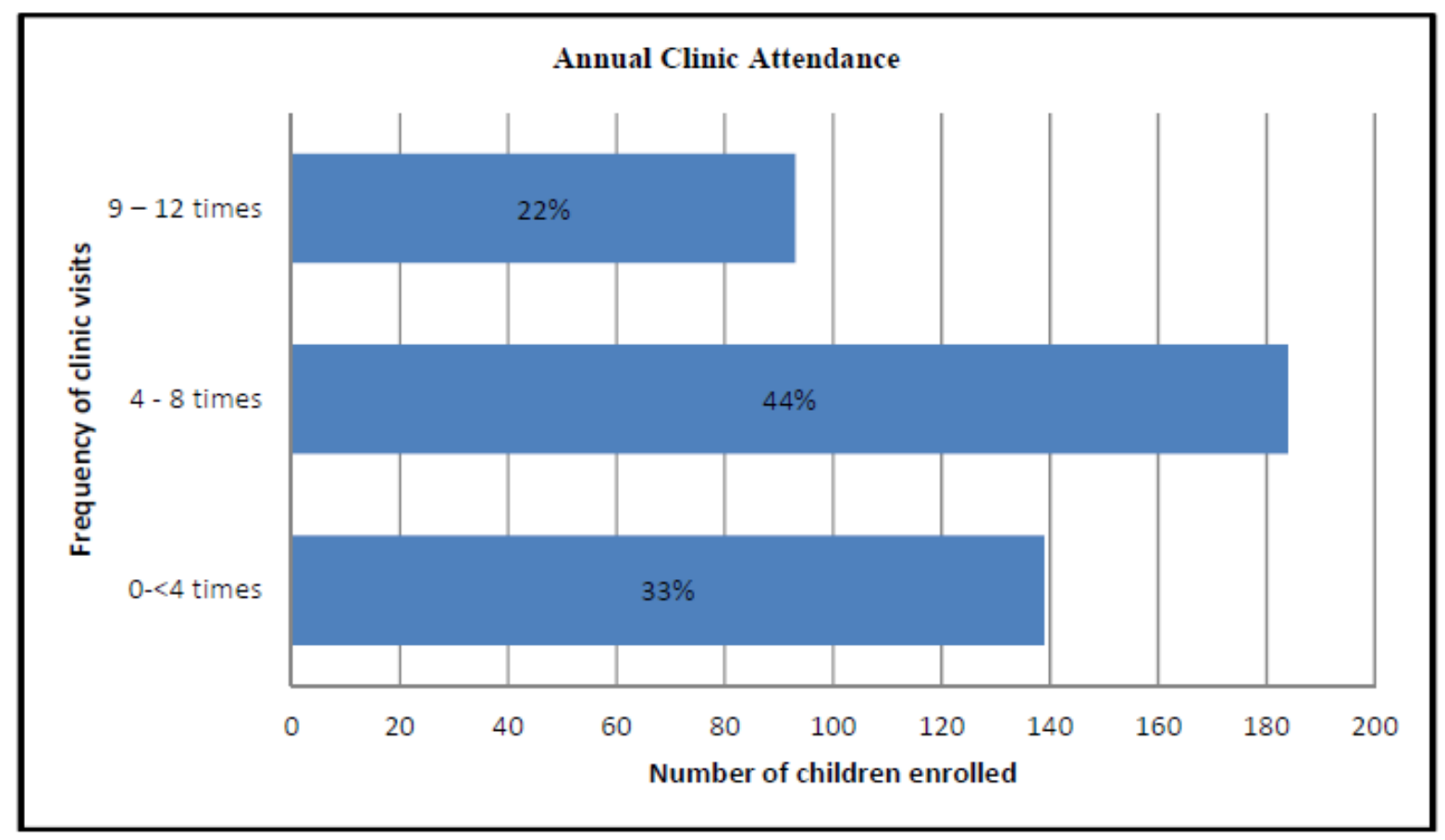

Figure 1. Bar chart illustrating annual clinic attendance among children less than 19 years living with HIV in a pediatric reference Hospital in Yaounde Cameroon. 
DOI: 10.21522/TIJPH.2013.05.04.Art020

ISSN: $2520-3134$

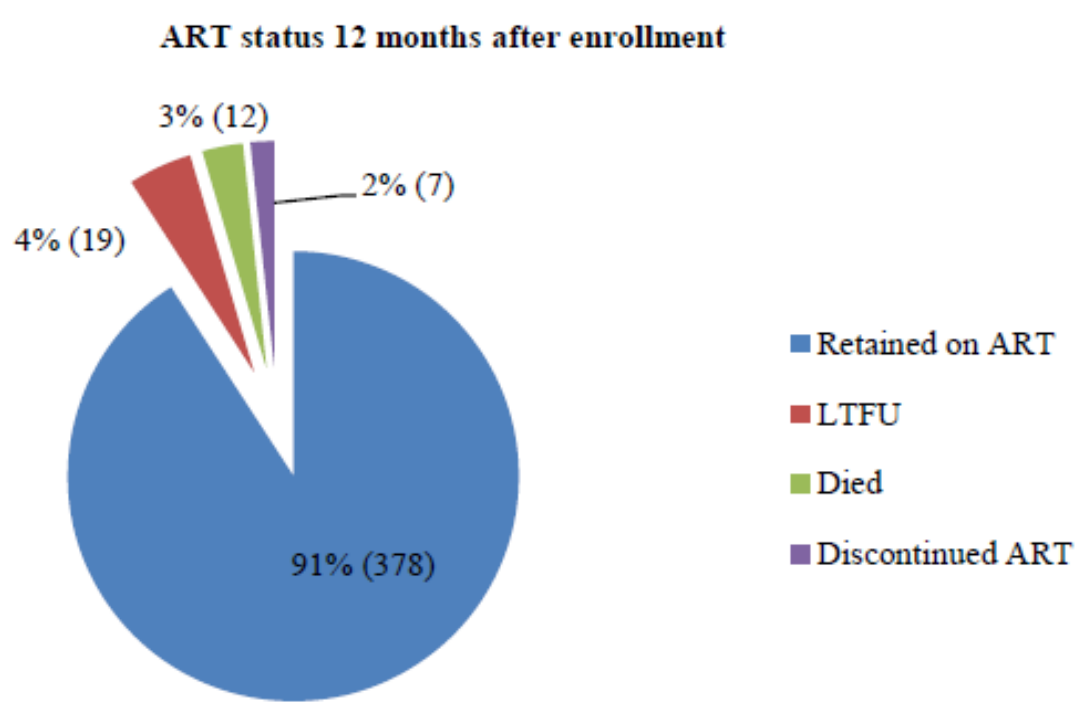

Figure 2. Pie chart to show distribution of retention rate among children less than 19 years living with HIV in a pediatric reference Hospital in Yaounde Cameroon.

Table 1. Demographic and baseline biological characteristics of children living with HIV less than 19 years assessed for 12- months retention in a pediatric Reference Hospital in Yaounde Cameroon

\begin{tabular}{|c|c|c|c|c|}
\hline \multirow[t]{2}{*}{ Characteristic } & \multirow[t]{2}{*}{ Enumerated } & \multirow[t]{2}{*}{ Excluded } & \multicolumn{2}{|c|}{ Total } \\
\hline & & & $\mathbf{N}$ & $\%$ \\
\hline \multicolumn{5}{|l|}{ Gender $(n=416)$} \\
\hline Female & 249 & 11 & 238 & 57,2 \\
\hline Male & 186 & 8 & 178 & 42,7 \\
\hline \multicolumn{5}{|c|}{ Age at initiation $(n=416)$} \\
\hline$<1 \mathrm{yr}$ & 53 & 4 & 49 & 11.8 \\
\hline $1-3 \mathrm{yrs}$ & 70 & 2 & 68 & 16.3 \\
\hline $3-5$ yrs & 84 & 4 & 80 & 19.2 \\
\hline $5-9$ yrs & 129 & 5 & 124 & 29.8 \\
\hline $9-15 \mathrm{yrs}$ & 66 & 3 & 69 & 16.6 \\
\hline $15-19$ yrs & 27 & 1 & 26 & 6.3 \\
\hline \multicolumn{5}{|c|}{ Caregiver $(n=394)$} \\
\hline Grandparents & 66 & 3 & 63 & 16.0 \\
\hline Parents & 294 & 14 & 280 & 71.1 \\
\hline Parent- relatives & 22 & 1 & 21 & 5.3 \\
\hline Siblings & 12 & 1 & 11 & 2.8 \\
\hline Missing & - & - & 19 & 4.8 \\
\hline \multicolumn{5}{|c|}{ Baseline CD4 $(n=373)$} \\
\hline CD4 \% & 169 & 13 & 156 & 41.8 \\
\hline CD4 count & 223 & 6 & 217 & 58.2 \\
\hline \multicolumn{5}{|c|}{ Illness at start $(n=416)$} \\
\hline Yes & 311 & 10 & 301 & 72.3 \\
\hline No & 125 & 10 & 115 & 27.7 \\
\hline
\end{tabular}


Texila International Journal of Public Health Volume 5, Issue 4, Dec 2017

Table 2. Retention per 13 monthly cohort of children less than 19 years living with HIV assessed for 12-months retention in a pediatric reference hospital in yaounde cameroon

\begin{tabular}{|c|l|l|l|l|}
\hline \multirow{2}{*}{ SN } & \multirow{2}{*}{ Month } & \multicolumn{2}{|l|}{ RETENTION } & \multirow{2}{*}{} \\
\cline { 3 - 4 } & & NUM & DEN & \\
\hline 1. & October 2015 & $\mathbf{3 7}$ & $\mathbf{3 3}$ & $\mathbf{8 9 \%}$ \\
\hline 2. & November 2015 & $\mathbf{3 4}$ & $\mathbf{3 0}$ & $\mathbf{8 8 \%}$ \\
\hline 3. & December 2015 & $\mathbf{3 5}$ & $\mathbf{2 3}$ & $\mathbf{7 7 \%}$ \\
\hline 4. & January 2016 & $\mathbf{3 6}$ & $\mathbf{3 6}$ & $\begin{array}{l}\mathbf{1 0 0} \\
\mathbf{\%}\end{array}$ \\
\hline 5. & February 2016 & $\mathbf{3 1}$ & $\mathbf{3 1}$ & $\begin{array}{l}\mathbf{1 0 0} \\
\mathbf{\%}\end{array}$ \\
\hline 6. & March 2016 & $\mathbf{3 2}$ & $\mathbf{3 0}$ & $\mathbf{9 4 \%}$ \\
\hline 7. & April 2016 & $\mathbf{2 6}$ & $\mathbf{2 6}$ & $\begin{array}{l}\mathbf{1 0 0} \\
\mathbf{\%}\end{array}$ \\
\hline 8. & May 2016 & $\mathbf{3 3}$ & $\mathbf{3 1}$ & $\mathbf{8 6 \%}$ \\
\hline 9. & June 2016 & $\mathbf{2 9}$ & $\mathbf{2 8}$ & $\mathbf{9 7 \%}$ \\
\hline 10. & July 2016 & $\mathbf{3 0}$ & $\mathbf{2 4}$ & $\mathbf{7 5 \%}$ \\
\hline 11. & August 2016 & $\mathbf{2 8}$ & $\mathbf{2 8}$ & $\mathbf{1 0 0}$ \\
\hline 12. & September 2016 & $\mathbf{3 1}$ & $\mathbf{2 9}$ & $\mathbf{9 4 \%}$ \\
\hline 13. & October 2016 & $\mathbf{3 4}$ & $\mathbf{2 9}$ & $\mathbf{8 5 \%}$ \\
\hline & TOTAL & $\mathbf{4 1 6}$ & $\mathbf{3 7 8}$ & $\mathbf{9 1 \%}$ \\
\hline
\end{tabular}

Table 3. Factors associated with retention in care among children less than 19 years living with HIV in a pediatric reference hospital in yaounde cameroon

\begin{tabular}{|l|l|l|}
\hline Characteristic & $\begin{array}{l}\mathrm{N}=416 \\
n(\%)\end{array}$ & $p$ value \\
\hline Gender $(\mathrm{n}=416)$ & & \\
\hline Female & $238(57.2)$ & 0.518 \\
\hline Male & $178(42.8)$ & 1.0 \\
\hline Age at initiation $(\mathrm{n}=416)$ & & \\
\hline$<1 \mathrm{yr}$ & $49(11.8)$ & 0.039 \\
\hline $1-3$ yrs & $68(16.3)$ & 0.941 \\
\hline 3-5 yrs & $80(19.2)$ & 0.305 \\
\hline 5-9 yrs & $124(29.8)$ & 0.365 \\
\hline 9-15 yrs & $69(16.6)$ & 0.401 \\
\hline $15-19$ yrs & $26(6.3)$ & 1.0 \\
\hline & & \\
Caregiver $(\mathrm{n}=394)$ & & \\
\hline Grandparents & $63(16.0)$ & 0.343 \\
\hline Parents & $280(71.1)$ & 0.132 \\
\hline Parent- relatives & $21(5.3)$ & 0.433 \\
\hline Siblings & $11(2.8)$ & 1.0 \\
\hline Baseline CD4 $(\mathrm{n}=373)$ & & \\
\hline CD4 \% & $156(41.8)$ & 0.049 \\
\hline CD4 count & $217(58.2)$ & 1.0 \\
\hline Illness at start $(\mathrm{n}=416)$ & & \\
\hline Yes & $301(72.3)$ & 0.864 \\
\hline
\end{tabular}


DOI: $10.21522 / \mathrm{TIJPH} .2013 .05 .04 . A r t 020$

ISSN: $2520-3134$

\begin{tabular}{|l|l|l|}
\hline No & $115(27.7)$ & 1.0 \\
\hline
\end{tabular}

\section{Acknowledgements}

We acknowledge the staffs that help with data extraction from registers at the reference pediatric center. Authors declare no competing interest.

\section{References}

[1].Abuogi LL, Smith C, McFarland EJ. Retention of HIV-Infected Children in the First 12 Months of AntiRetroviral Therapy and Predictors of Attrition in Resource Limited Settings: A Systematic Review. PLoS ONE (2016)11(6): e0156506. doi: 10.1371/ journal.pone.0156506.

[2].Alvarez-Uria G, Pakam R, Midde M, Naik PK. Entry, Retention, Virological Suppression in an HIV Cohort Study in India: Description of the Cascade of Care and Implications for Reducing -Related Mortality in Lowand Middle-Income Countries. Interdisciplinary Perspectives on Infectious Diseases. 2013; Article ID 384805: 8. PubMed | Google Scholar.

[3].Amuron B, Namara G, Birungi J, Nabiryo C, Levin J, Grosskurth H, et al. Mortality and loss-to-follow-up during the pre-treatment period in an antiretroviral therapy programme under normal health service conditions in Uganda. BMC Public Health. 2009; 9 (1):290.

[4].Atemnkeng FN et al. Cause of poor retention in care in adult HIV patients on treatment at the Bafoussam Regional Hospital, Cameroon. STD-AIDS-2015 .Conference series LLC [Internet]. [Cited 2016 Oct 26]. Available from: http://hiv-aids std. conferenceseries.com /abstract /2015/cause-of-poor-retention-in-care-inadult-hiv-patients-on-treatment-at-the-bafoussam-regional-hospital-cameroon.

[5].Bassett IV, Regan S, Chetty S, Giddy J, Uhler LM, Holst H, Ross D, Katz JN, Walensky RP, Freedberg KA, Losina E. Who starts antiretroviral therapy in Durban, South Africa? not everyone who should. AIDS. 2010; 24 (Suppl 1):S37-44. PubMed | Google Scholar.

[6].Boyles TH, Wilkinson LS, Leisegang R, Maartens G. Factors influencing retention in care after starting antiretroviral therapy in a rural South African programme. PloS One. 2011 May 3; 6(5):e19201.

[7].Charurat M, Oyegunle M, Benjamin R, Habib A, Eze E, Ele P, Ibanga I, Ajayi S, Eng M, Mondal P, Gebi U, Iwu E, Etiebet M, Abimiku A, Dakum P, Farley J, Blattner W. Patient Retention and Adherence to Antiretrovirals in a Large Antiretroviral Therapy Program in Nigeria: A Longitudinal Analysis for Risk Factors. PLoS ONE. 2010; 5(5): 10584. PubMed | Google Scholar.

[8].Dahab M, Charalambous S, Hamilton R, Fielding K, Kielmann K, Churchyard GJ, et al. "That is why I stopped the ART": Patients' \& providers' perspectives on barriers to and enablers of HIV treatment adherence in a South African workplace programme. BMC Public Health. 2008; 8:63.

[9].Fox MP, Rosen S. Patient retention in antiretroviral therapy programs up to three years on treatment in subSaharan Africa, 2007-2009: systematic review. Trop Med Int Health TM IH. 2010 Jun; 15 Suppl 1:1-15.

[10]. Forster M, Bailey C, Brinkhof MW, Graber C, Boulle A, Spohr M, Balestre E, May M, Keiser O, Jahn A, Egger M. Electronic medical record systems, data quality and loss to follow-up: survey of antiretroviral therapy programmes in resource-limited settings. Bull World Health Organ. 2008; 86:939-947. PubMed | Google Scholar.

[11]. Frambo B. A. A, Diko A C, Kamga D, Bieme-Ndi E, Ako-Arrey D, Epanya N, Sadate-Ngatchou P, Abessouguie I, Nzuobontane D. Retention in HIV Care and Treatment, 12 months after Initiation on ART. 10th International Workshop on HIV treatment, Pathogenesis and Prevention Research in Resource-Limited settings, May 2016, Abstract Book.

[12]. Jarett O, Mwamburi M. 5th IAS Conference on HIV Pathogenesis, Treatment, and Prevention [Internet]. Medscape. [cited 2016 Oct 28]. Available from: www.medscape.com/viewcollection/30399. Accessed 21 February 2017.

[13]. Lessells RJ, Mutevedzi PC, Cooke GS, Newell ML. Retention in HIV care for individuals not yet eligible for antiretroviral therapy: rural Kwazulu-Natal, South Africa. Journal of Acquired Immune Deficiency Syndromes. 2011; 56(3):79-86. PubMed |Google Scholar. 
[14]. Mberi MN, Kuonza LR, Dube NM, Nattey C, Manda S, Summers R. Determinants of loss to follow-up in patients on antiretroviral treatment, South Africa, 2004-2012: a cohort study. BMC Health Serv Res. 2015 Jul 4; 15:259.

[15]. Muula AS, Ngulube TJ, Siziya S, Makupe CM, Umar E, Prozesky HW, Wiysonge CS, Mataya RH. Gender distribution of adult patients on highly active antiretroviral therapy (HAART) in Southern Africa: a systematic review. BMC Public Health. 2007; 7: 63. PubMed | Google Scholar.

[16]. National AIDS Control Organisation (NACO). India HIV Estimates. 2011. Available from http://naco.gov.in/upload/Surveillance/Reports\%20\&\%20Publication/HSS\%202010-

11_Technical\%20Brief_30\%20Nov\%2012.pdf (Accessed 29th May 2017). Google Scholar.

[17]. Remien RH, Chowdhury J, Mokhbat JE, Soliman C, Adawy ME, Sadr WE. Gender and care: access to HIV testing, care, and treatment. J Acquir Immune Defic Syndr. 2009; 51(Suppl 3):S106-110. PubMed | Google Scholar.

[18]. Rosen S, Fox MP, Gill CJ. Patient retention in antiretroviral therapy programs in Sub-Saharan Africa: a systematic review. PLoS Med. 2007; 4(10):e298. PubMed | Google Scholar.

[19]. Roura M, Busza J, Wringe A, Mbata D, Urassa M, Zaba B. Barriers to sustaining antiretroviral treatment in Kisesa, Tanzania: a follow-up study to understand attrition from the antiretroviral program. AIDS Patient Care STDs. 2009 Mar; 23(3):203-10.

[20]. Sengayi M, Dwane N, Marinda E, Sipambo N, Fairlie L, Moultrie H. Predictors of loss to follow-up among children in the first and second years of antiretroviral treatment in Johannesburg, South Africa. Global health action. 2013; 6:19248. doi: 10.3402/gha.v6i0.19248 PMID: 23364098.

[21]. Sherer R, Stieglitz K, Narra J, Jasek J, Green L, Moore B, et al. HIV multidisciplinary teams work: support services improve access to and retention in HIV primary care. AIDS Care. 2002 Aug; 14 Suppl 1:S31-44.

[22]. Thida A, Tun STT, Zaw SKK, Lover AA, Cavailler P, Chunn J, et al. Retention and risk factors for attrition in a large public health ART program in Myanmar: a retrospective cohort analysis. PloS One. 2014; 9(9):e108615.

[23]. Ulett KB, Willig JH, Lin H-Y, Routman JS, Abroms S, Allison J, et al. The therapeutic implications of timely linkage and early retention in HIV care. AIDS Patient Care STDs. 2009 Jan; 23(1):41-9.

[24]. WHO | Guidelines: HIV. WHO. [cited 2016 Oct 28]. Available from: http://www.who.int/hiv/pub/guidelines/en/ Accessed 16 March 2017.

[25]. WHO. UNAIDS World AIDS Day Report 2012 Core epidemiology slides. 2012 Available from: http://www.who.int/hiv/data/en/ (Accessed 29 May 2017).Google Scholar. 